\title{
Acute Myeloid Leukemia: Clinical Spectrum of 125 Patients
}

\author{
Sadia Sultan ${ }^{1 *}$, Hasan Abbas Zaheer ${ }^{2}$, Syed Mohammed Irfan¹, Sana Ashar ${ }^{1}$
}

\begin{abstract}
Background: Acute myeloid leukemia is an acquired clonal heterogeneous stem cell disorder. Hence, various parameters are sought out to categorize this disease into subtypes, so that as a consequence specific treatment modalities can be offered. Conventionally, the practically used method for classification utilizes French American British (FAB) criteria based on morphology and cytochemistry. The aim of present study was to determine the current spectrum of AML sub types in patients in Karachi. Materials and Methods: This single centre cross sectional study was conducted at Liaquat National Hospital, Karachi, extending from January 2010 to December 2014. Data were retrieved from archives were analyzed with SPSS version 22. Results: A total of 125 patients were diagnosed at our institution with de novo AML during five years period, 76 males and 49 females. Median age was 34.5 years. AML-M1 was the predominant FAB subtype (23.2\%) followed by M2 (18.4\%), M3 and M4 (16\% each), M0 (14.4\%), M5 (7.2\%), M6 (3.2\%) and M7 (1.6\%). Conclusions: AML in Pakistani patients is seen in a relatively young population. The most common FAB subtype observed in our study was acute myeloblastic leukemia, without maturation (M1).
\end{abstract}

Keywords: Acute myeloid leukemia - FAB subtypes - Karachi, Pakistan

Asian Pac J Cancer Prev, 17 (1), 369-372

\section{Introduction}

Acute myeloid leukemia (AML) represents one of the most frequent, biologically and clinically diverse disorder amongst all hematopoietic malignancies. (Zhou et al., 2013; Ahmad et al., 2014). In the literature annual incidence for AML is 3-4/100,000 individuals each year (Schlenk., 2014). The incidence of AML increases gradually with age and median age of acute myeloid leukemia's patients is around 70 years internationally (Ossenkoppele and Löwenberg., 2015).

The disease biology among AML patients is highly heterogeneous (Yang et al., 2012; Su et al., 2014). Therefore certain parameters are needed to classify this disease into biologic entities to understand its pathogenesis and more importantly to determined treatment modality (Tien et al., 1995; Schoch and Haferlach., 2002).

French-American-British classification for acute myeloid leukemia had been widely accepted and applicable due to its ease and good reproducibility. Conversely, the obligation of karyotyping and immunophenotyping in WHO classification makes it difficult for developing countries like Pakistan to put this classification in routine practices. FAB classification is purely based on morphologic and cytochemical features; as they do not require advanced technology and can be easily applied in most laboratories (McKenna., 2000). It mainly categorizes according to their lineage differentiation and degree of maturation of the leukemic cells. In view of these merit, the FAB classification was implemented nationally and internationally. Previous study, reported from Pakistan was determined AML-M2 as the predominant variant in adults AML (Harani et al., 2005).

Present study is design to determine the current spectrum of AML subtypes in our population. As the patients from all over the city belonging to diverse racial groups were come to our centrally located tertiary care center, thus our study tends to establish an existing trend of AML locally.

\section{Materials and Methods}

This is a retrospective cross sectional analysis conducted at hematology department of Liaquat National Hospital. The study was extended over a period of 5 years from January 2010 to December 2014.

Socio-demographic data including age, gender and contact numbers were recorded. All patients underwent detailed history, general physical and systemic examination. Patients having history of prior hematological disorders like Myelodysplastic syndrome, Myeloproliferative neoplasm, Myelodysplastic/ Myeloproliferative entity or undifferentiated leukemia and cases of pediatrics AML were excluded from the analysis. The patients who were known cases of secondary AML were also not included. A total of 125 adults ( $\geq 15$ years) with newly diagnosed 
untreated de novo AML were integrated in the study. The diagnosis of AML was established according to the customary FAB criteria, and was based on bone marrow morphology and cytochemical staining (Bennett et al., 1985).

Complete blood counts were done on Cell Dyne automated counter (Abbott, USA). All peripheral blood smears were reviewed by specialist hematopathologists. Bone marrow aspiration was done from posterior iliac crest through Jamshidi needle and was stained by Leishman's stain. Cytochemical stains were carried out on each bone marrow smears including Sudan Black B (SBB), Periodic acid-Schiff (PAS) and Alpha naphthyl acetate esterase by commercially provided kits from Merck Diagnostic, as per manufacturer's instructions. Each test results were validated by running simultaneously appropriate positive controls. Immunophenotyping was done where it was deemed necessary, in patients with diagnostic uncertainty.

Approval from the institutional ethical and research review committee was obtained prior to the study.

\section{Data analysis}

The demographic data, clinical characteristics, and laboratory results were analyzed by descriptive analysis. Data was compiled and analyzed using the Statistical Package for the Social Sciences version 22.0 (SPSS Inc, Chicago, IL, USA). The results were expressed as mean $\pm \mathrm{SD}$ for quantitative variables and qualitative variables are presented as frequency \& percentages.

\section{Results}

Out of 125 patients, 76 were males $(60.8 \%)$ and 49 were females (39.2\%) with male to female ratio of 1.5:1. Age ranged between 15 and 85 years with a mean age of

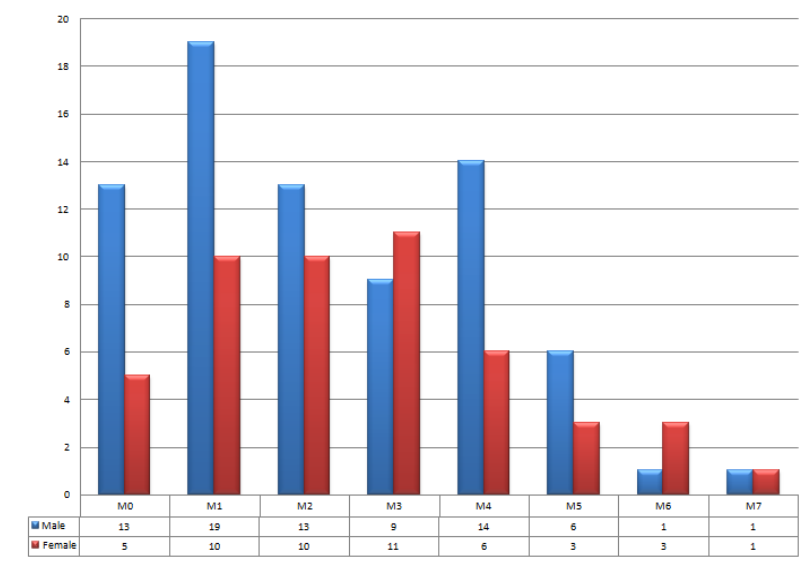

Figure 1. Spectrum of FAB Classification according to Gender
$37.97 \pm 20.12$ years and median age of 34.5 years. Overall $95(76 \%)$ patients were under 50 years of age, and only $30(24 \%)$ patients were above 50 years.

The major complaints were fever in $91(72.8 \%)$ patients; generalized weakness in $75(60 \%)$ patients; bleeding in $47(37.6 \%)$ patients and dyspnea in 15 $(12 \%)$ patients. Physical examination revealed pallor as a predominant finding detected in $71(56.8 \%)$ patients followed splenomegaly and hepatomegaly in 20 (16\%) and $16(12.8 \%)$ patients respectively. Lymphodenopathy was noted in $13(10.4 \%)$ patients.

The mean hemoglobin was $8.19 \pm 2.12 \mathrm{~g} / \mathrm{dl}$ with the mean MCV of $85.98 \pm 9.83 \mathrm{fl}$. The mean total leukocyte count of $43.08 \pm 68.45 \times 10^{9} / 1$; Absolute neutrophilic count (ANC) of $3.09 \pm 6.66 \times 10^{9} / 1$ and the mean platelets count were $62.32 \pm 78.61 \times 10^{9} / 1$.

Anemia was noted in $102(81.6 \%)$ patients. Thrombocytopenia (platelets count $<100 \times 10^{9} / 1$ ) was detected in $105(84 \%)$ patients.

We found Acute myeloblastic leukemia, without maturation (AML-M1) to be the commonest subtype comprising of 29 out of 125 cases $(23.2 \%)$ followed by AML-M2 in $23(18.4 \%)$ patients, M3 \& M4 variants in 20 patients each (16\%), M0 subtype seen in $18(14.4 \%)$ patients, while M5 was noted in $9(7.2 \%)$ patients. The least encountered types were M6 and M7 detected in 4 (3.2\%) and $2(1.6 \%)$ patients respectively. Comparative analyses with other regional studies are shown in table-1.

In relation to gender distribution males patients revealed predominance of AML-M1 type while female patients show AML-M3 as a frequent variant (figure1).

\section{Discussion}

AML is a disease of the elder, with a median age of around $\sim 70$ years (Juliusson et al., 2009). Surprisingly, the median age of the patients in our study is 34.5 years. When compared with international reports, our finding is in distinction with studies published from Sweden and Germany, where the median age were 71 and 60 years respectively. (Lazarevic, 2014; Pastore, 2014). However, some studies from our part earlier reported low median age (Kakepoto et al., 2002; Harani et al., 2005). Conceivably this variance may be elucidated by difference based on geographical and genetic makeup between two racial groups and also accountable is the high mean ages in western countries compared to east.

There are two standard classification systems that are widely used for the diagnosis of acute myeloid leukemia (AML). The French-American-British (FAB) classification system is based on differentiation and

Table 1. FAB Classification of Adults AML in Prior Pakistani Studies

\begin{tabular}{|c|c|c|c|c|c|c|c|c|}
\hline Author/year & M0 & M1 & M2 & M3 & M4 & M5 & M6 & M7 \\
\hline Hassan et al., $1993(n=62)$ & 1.6 & 22.5 & 32.2 & 9.1 & 22.5 & 8.6 & 1.6 & 1.6 \\
\hline Chaudry et al., $1993(n=54)$ & 0 & 13 & 44.4 & 11.1 & 24 & 3.7 & 3.7 & 0 \\
\hline Kakepoto et al., $2002(n=74)$ & 0 & 8.1 & 16 & 15 & 46 & 9.5 & 0 & 2.7 \\
\hline Harani et al., $2005(n=95)$ & 1.2 & 9.4 & 30.5 & 12.6 & 36.8 & 6.3 & 0 & 0 \\
\hline Asif et al., $2013(n=56)$ & 1.8 & 32.1 & 14.3 & 19.6 & 19.6 & 9.0 & 1.8 & 1.8 \\
\hline Ali et al., $2013(n=100)$ & NR & 33 & 26 & 4 & 13 & 9 & 5 & NR \\
\hline Present study $2015(n=125)$ & 14.4 & 23.2 & 18.4 & 16 & 16 & 7.2 & 3.2 & 1.6 \\
\hline
\end{tabular}


morphological grounds to define specific types. Whilst World Health Organization (WHO) classification are mainly reliant on immunophenotyping and conventional karyotype. FAB classification has been widely adopted in most of haematology laboratories of Pakistan.

The FAB classification of AML has been comprehensively studied at both national and international levels (Kakepoto et al., 2002; Asif et al., 2013; Walter et al., 2013). However there is a breach of a decade between prior regional (Karachi) studies and in the present study. The present study has illustrated the existing spectrum of FAB classification in Pakistani adults AML patients. We determined AML-M1 as the commonest type, followed by $\mathrm{M} 2$ and M3, M4 and M0 respectively. This distribution of various FAB types was partly comparable with the prior studies. Nevertheless more pertinent finding of the present study is changeover from M2 subtype towards M1 variant.

Table-1 depicted the FAB distribution in various prior studies on Pakistani patients with adult's AML. Most published data indicate the predominance of M2 (1990) and M4 (2005) as a common subtypes previously (Chaudhry et al., 1993; Hassan et al., 1993; Harani et al., 2005). However, the M1 dominance was seen in the present study which is in concurrence with the recent studies by Asif and Ali et al from Pakistan (Asif et al., 2013; Ali et al., 2013). Similarly, Hamayun et al from northern area also revealed M1 as predominant subtype accountable in $50 \%$ of their study subjects (Hamayun et al., 2005). Nevertheless it seems to have a gradual transition from AML-M2 (30-89\% blast cells) towards a more advanced AML-M1 (blast cell $>90 \%$ ) disease phenotype.

When compared these findings with recent Indian study on a large cohort $(\mathrm{n}=209)$, the predominant subtypes were M5 (25.4\%) and M2 (23.9\%) (Sarojam et al., 2014). Studies from Saudi Kingdom reported the predominance of M4 and M5 variants (Harakati et al., 1993; Spence et al., 1988). Nakase et al showed AML-M4 as common subtype in Australian AML patients compared to Japanese, where AML-M2 is frequent (Nakase et al., 2000). However recently one study on largest cohort $(n=5848)$ of patients from USA disclosed M1 in 25\% of AML patients (Walter et al., 2013). Present study also confirms M1 (23.2\%) as the most common type followed by M2.

The differences in AML subtypes may be due to the subjectivity of morphological diagnosis, however some genetic factors may be responsible for a particular FAB subtype of AML in our population. Notably most studies at national level had small number of patients and perhaps with under exploitation of cytochemical stains. Besides these studies might not have utilized immunophenotyping that resulted in an inaccurate diagnosis.

A number of biological and clinical factors affect the outcome and response to treatment in patients with AML. The differences in prognosis have been observed among the different FAB subtypes (Asif et al., 2013). Generally cases of M5, M6, M0 and M7 have a worse prognosis than those of M2, M3 and M4 (Asif et al., 2013). It is also note worthy, that substantiation of maturation in leukemic cells, presence of granules or Auer rods and positivity of Sudan Black Band reactions are associated with a more favorable prognosis (Asif et al., 2013). In our study most common subtype was AML-M1 which lack maturation, might points towards aggressive phenotype and poor prognosis. This may also represent high disease burden in our patients could have been due to the late presentation.

Clinically, an AML patient with an increase percentage of leukemic blasts tends to have FLT3-ITD mutation (Gregory et al, 2009). Recently Mawali et al showed that half of the patients with M1-FAB subtype had FLT3/ITD positivity which has been associated with poor prognosis (Mawali et al., 2013). However we did not evaluated mutational status in our patients which is our limitation. But perhaps it would be same in our population, which needs validation.

The limitations of the study need to be mentioned; firstly karyotyping analysis was not performed, as this facility was not available at our institute. Secondly informative markers including molecular testing and mutational status were also not determined due to financial constrains.

In conclusion, AML is predominantly seen in very younger age group in Pakistan. Though, previously other national studies had reported AML-M2 as a frequent type, but present study revealed Acute myeloblastic leukemia, without maturation (M1) as a major subtype. Prospective studies should be pursueded on larger patient series to incorporate novel molecular markers and its prognostication in the local population.

\section{References}

Ali A, Siddique MK, Shakoori AR (2013). Frequency of NPM1 mutations in pakistani acute myeloid leukemia patients. Pakistan J Zool, 45, 833-41.

Ahmad F, Mohota R, Sanap S, et al (2014). Molecular evaluation of DNMT3A and IDH1/2 gene mutation: frequency, distribution pattern and associations with additional molecular markers in normal karyotype Indian acute myeloid leukemia patients. Asian Pac J Cancer Prev, 15, 1247-53.

Asif N, Hassan K (2013). Acute myeloid leukemia amongst adults. Journal of Islamabad Medical \& Dental College, 2, 58-63.

Bennett JM, Catovsky D, Daniel MT, et al (1985). Proposed revised criteria for the classification of acute myeloid leukemia. A report of the French-American-British Cooperative Group. Ann Intern Med, 103, 620-5.

Chaudhry MT, Tayyab M, Faooqi IA (1993). Acute nonlymphoblastic leukemia in adults. J Pak Med Assoc, 43, 259-61.

Gregory T, Wald D, Chen Y (2009). Molecular prognostic markers for adult acute myeloid leukemia with normal cytogenetics. Journal of Hematology \& Oncology, 2, 23.

Hassan K, Qureshi M, Shafi S, et al (1993). Acute myeloid leukaemia- FAB classification and its correlation with clinico- heamatological features. J Pak Med Assoc, 43, 200-3.

Harani MS, Adil SN, Shaikh MU, et al (2005). Frequency of fab subtypes in acute myeloid leukemia patients at Aga Khan University Hospital Karachi. J Ayub Med Coll Abbottabad, 17, 26-9.

Hamayun M, Khan SA, Muhammad W (2005). Investigation on the prevalence of leukemia in north west frontier province of Pakistan. Turkish journal of cancer, 35, 119-22.

Harakati MS, Al-Momen AM, Ajarim DS, et al (1998). Adult 
acute myeloblastic leukemia: Experience at King Khalid University Hospital. Ann Saudi Med, 18, 221-5.

Juliusson G, Antunovic P, Derolf A, et al (2009). Age and acute myeloid leukemia: real world data on decision to treat and outcomes from the Swedish Acute Leukemia Registry. Blood, 113, 4179-87.

Kakepoto GN, Burney IA, Zaki S, et al (2002). Long-term outcomes of acute myeloid leukemia in adults in Pakistan. J Pak Med Assoc, 52, 482-6.

Lazarevic V, Hörstedt AS, Johansson B, et al (2014). Incidence and prognostic significance of karyotypic subgroups in older patients with acute myeloid leukemia: the Swedish population-based experience. Blood Cancer J, 28, 188.

Mawali A, Gillis D, Lewis L (2013). Characteristics and Prognosis of Adult Acute Myeloid Leukemia with Internal Tandem Duplication in the FLT3 Gene. Oman Med J, 28, 432-40.

McKenna RW (2000). Multifaceted approach to the diagnosis and classification of acute leukemias. Clin Chem, 46, 1252-9.

Nakase K, Bradstock K, Sartor M, et al (2000). Geographic heterogeneity of cellular characteristics of acute myeloid leukemia: a comparative study of Australian and Japanese adult cases. Leukemia, 14, 163-8.

Ossenkoppele G, Löwenberg B (2015). How I treat the older patient with acute myeloid leukemia. Blood, 125, 767-74.

Pastore F, Dufour A, Benthaus T, et al (2014). Combined molecular and clinical prognostic index for relapse and survival in cytogenetically normal acute myeloid leukemia. $J$ Clin Oncol, 20, 1586-94.

Sarojam S, Vijay S, Raveendran S (2014). FLT3 Mutation as a Significant Prognostic Marker in de novo Acute myeloid leukemia patients: Incidence, distribution and association of cytogenetics findings in South India. Middle East Journal Cancer, 5, 185-96.

Schlenk RF (2014). Post-remission therapy for acute myeloid leukemia. Haematologica, 99, 1663-70.

Schoch C, Haferlach T (2002). Cytogenetics in acute myeloid leukemia. Curr Oncol Rep , 4, 390-7.

Spence DG, Roberts GT, De Vol EB, et al (1988). Acute myeloid leukemia in Saudia Arabia: Morphologic classification using FAB subgroups. Ann Saudi Med, 8, 179-84.

Su L, Li X, Gao SJ, et al (2014). Cytogenetic and genetic mutation features of de novo acute myeloid leukemia in elderly Chinese patients. Asian Pac J Cancer Prev, 15, 895-8.

Tien HF, Wang CH, Lin MT, et al (1995). Correlation of cytogenetic results with immunophenotype, genotype, chemical features and ras mutation in acute myeloid leukemia: a study of 235 Chinese patients in Taiwan. Cancer Genet Cytogenet, 84, 60-8.

Walter RB, Othus M, Burnett AK, et al (2013). Significance of FAB subclassification of "acute myeloid leukemia, NOS" in the 2008 WHO classification: analysis of 5848 newly diagnosed patients. Blood, 121, 2424-31.

Yang XF, Sun AN, Yin J, et al (2012). Monosomal karyotypes among 1147 Chinese patients with acute myeloid leukemia: prevalence, features and prognostic impact. Asian Pac J Cancer Prev, 13, 5421-6.

Zhou L, Zhu YY, Zhang XD, et al (2013). Risk effects of GST gene polymorphisms in patients with acute myeloid leukemia: a prospective study. Asian Pac J Cancer Prev, 14, 3861-4. 